\title{
Die Versorgungslücke droht
}

unattraktiv werden lassen. Budgetierung und wenig sinnvolle Umstrukturierungen - wie z. B. die Aut-idem-Verordnung - erschweren den Praxisalltag.

Die Zahl der Medizinstudenten hat sich in den letzten Immer mehr Kollegen würden sieben Jahren um 4,5\% verringert. Studienabbrecher und Queraussteiger machen inzwischen rund $20 \%$ eines Studienjahrganges aus. Der ärztliche Nachwuchs bricht weg. Eine Versorgungslücke droht - auch in der Allergologie.

D er $\mathrm{A} D A$ hat in den vergangenen Jahren immer wieder auf bestehende Mängel bei der Diagnostik, Therapie, Prävention und Rehabilitation allergischer Erkrankungen hingewiesen. Qualitätsorientierte Konzepte zur Besserung der Situation wurden erarbeitet und präsentiert. Eine Umsetzung durch die zuständigen Gremien erfolgte aus den verschiedensten Gründen bis heute nicht. Ausführlich ist im Weißbuch „Allprä- 